\title{
Could Ultrasound Inactivate COVID-19 in the Environment and in the Air, Thus Preventing the Spread of the Pandemic?
}

\author{
Sandro Norelli* \\ Department of Environment and Health, Istituto Superiore di Sanità, Rome, Italy
}

*Corresponding author: Sandro Norelli, Department of Environment and Health, Istituto Superiore di Sanità, Rome, Italy; E-mail: sandro.norelli@iss.it

Received: July 28, 2021; Accepted: August 11, 2021; Published: August 20, 2021

\begin{abstract}
The novel severe acute respiratory syndrome coronavirus 2 (SARS-CoV-2), also called COVID-19, started by Wuhan, China, in 2019, has caused a pandemic which has quickly involved the entire world and raised public health concerns. The scientific community is actively exploring treatments that would potentially be effective in combating COVID-19. Viral infections, especially those that are transmitted by air, are the most contagious infectious diseases and cause major biological, clinical and socioeconomic problems worldwide. COVID-19 spreads in the air and therefore rapidly contagious, thus finding appropriate and timely treatment to cure the entire population and above all to prevent transmission between individuals, is extremely necessary in a short time. Considerable efforts are being made to seek therapy on the infected human being, in fact several drugs already used in the past for SARS or HIV-1 are tested, in what is called a drug repositioning or label off strategy, but it is very important to prevent contagion before other individuals are infected, and especially the most fragile ones, even to decongest the structures of the health system also to avoid neglecting other pathologies as unfortunately is happening in this pandemic period. High intensity ultrasound is becoming important and more widely used in the food industry for microorganisms decontamination and is one of the new technologies that have been suggested as an alternative to current heat treatments for microbial inactivation, including viruses. Also for synergistically enhanced elimination of organic pollutants and pathogenic microorganisms from water since the early 2000s, dual-frequency ultrasound has received much attention. Here, my hypothesis consist to use ultrasound, as a valid prevention clean mean, which propagates well in the air, such as respiratory viruses, to fight the COVID-19 expansion in the air environment, and thus block the transmission from one individual to another, especially indoors and also from objects to individuals. Ultrasoud are also used in the Protein misfolding cyclic amplification (PMCA) technique used to amplify prions. The growing chain of misfolded protein is then blasted with ultrasound by sonication, breaking it down into smaller chains and so rapidly increasing the amount of abnormal protein available to cause conversions. Ultrasounds are waves with frequencies between $20 \mathrm{kHz}$ and $20 \mathrm{MHz}$, well known have effects on molecular structures of various microorganisms, even viruses, but are not harmful to humans, and so could potentially damage also the new Coronavirus, COVID-19 especially by damaging the superficial spike S-glycoprotein that the virus uses to enter cells and infect them, and block or slow down the epidemic which is destroying many human lives. Experimentally, COVID-19 and its variants, can be treated with ultrasonic waves at different exposure times, then Vero E6 cells (African green monkey kidney cells) permissive to the SARS-CoV-2, are infected in vitro with the treated virus with ultrasounds and tested for efficacy of on the suppression of activity of COVID-19 and therefore for the decrease or block of infectivity. After a possible positive outcome of the laboratory experimentation, ultrasounds can be applied in closed environments frequented by people including homes, offices, supermarkets, schools, buses, subways to purify the air and avoid the spread of contagion.
\end{abstract}

Keywords: COVID-19, Virology, Ultrasound, Enviroments, Prevention

\section{Introduction}

The COVID-19 pandemic represents the greatest global public health crisis since the pandemic influenza outbreak of 1918, a bit more than a century ago. The novel coronavirus disease 2019 (COVID-19) has resulted in the deaths of more than 248000 persons worldwide as of May 4, 2020, on the same date in New York more than 19400 individuals have died [1]. Research is under way to identify vaccines and therapeutics for COVID-19, including repurposing of medications. Facing a new and unknown virus, antiviral agents previously used to treat other infections such as SARS and Middle East Respiratory Syndrome (MERS), have been considered as the first potential candidates for firstline therapy to treat COVID-19. Chloroquine and hydroxychloroquine, old drugs used in the treatment of malaria and inhibitors of protease of HIV-1 have been previously studied, where evidence of efficacy has been found and they have anti coronavirus characteristics in vitro $[2,3]$. The findings support the insight that chloroquine/hydroxychloroquine have efficacy in the treatment of COVID-19 [4]. Therefore, based on evidence from in vitro studies on the suppression of activity of severe acute respiratory syndrome coronavirus 2 (SARS-CoV-2) and other coronavirus strains, interest increased in the use of hydroxychloroquine and chloroquine with the possible addition of azithromycin for the treatment of COVID-19 [5,6]. At the same time, a great number of clinical trials have been launched to investigate the potential efficacy therapies for COVID-19 highlighting the urgent need to get as quickly as possible high-quality evidence. This new virus, COVID-19, such 
as respiratory viruses, spreads in the air, and therefore also rapidly contagious therefore, finding effective and timely treatment to cure the entire population or better still, prevent contagion, is a very difficult task. For so much we must find alternative and effective methods also for the prevention of global spread as well as for the cure in order not to collapse the health system.

\section{Hypothesis}

My hypothesis here consist to use ultrasound, as a valid prevention mean, which propagates well in the air, to fight the SARSCoV-2 in environments, especially indoors, and it is well known have effects on molecular structures of various microorganisms, including viruses, but they are not harmful to humans. Ultrasound is one of the new technologies that have been suggested as an alternative to current heat treatments for microbial inactivation [7,8]. Ultrasound is defined as a sonic wave at frequencies over the threshold human hearing. Ultrasonic waves are generally classified by their frequency and their wavelength. Waves with frequencies between 20 and $40 \mathrm{kHz}$ are considered as high-energy or high-power ultrasound Figure 1 [8].

High intensity ultrasound is becoming important and more widely used in the food industry for microorganisms decontamination. This sterilization technique has been evaluated to improve food safety and to replace common processing with chemical additive compounds. The efficiency of a horn-type power ultrasound treatment $(300 \mathrm{~W}$ and $600 \mathrm{~W}, 28 \mathrm{kHz}, 10-30 \mathrm{~min}$ ) on Listeria monocytogenes, Bacillus cereus, Escherichia coli, Salmonella typhimurium bacteria suspensions and phytoviruses was examined in this study [9]. The results of this study showed that ultrasonic treatment can be used to eliminate vegetative cells of gram-positive and gram-negative bacteria from 1.59 to $3.4 \mathrm{log}$ in bacterial suspensions and some phytoviruses in fruits [9]. Also for treatment and disinfection of water dual-frequency ultrasound since the early 2000s, dual-frequency ultrasound (DFUS) has received much interest [10]. Elimination of organic pollutants and pathogenic microorganisms from water occurs indirectly by generating reactive oxygen species (ROS) induced through exposure the water to DFUS [10]. The nonlinear dynamics of microbubbles upon DFUS exposure produces additional frequencies, such as harmonics, subharmonics, ultraharmonics and combination frequencies. These increase the probability of bubbles collapse, thereby enhancing cavitation and generating more reactive oxygen species for advanced oxidation processes (AOPs) [10]. The protein misfolding cyclic amplification (PMCA) mimics in vitro the process of prion propagation, which occurs in vivo [11]. PMCA requires the incubation of prions with an excess of $\operatorname{Pr}(\mathrm{PC})$ (a normal glycophosphatidyl-anchored glycoprotein) in a test tube, which is placed in a dedicated sonicator and subjected to a process of cyclically repeated phases of sonication and incubation. Brains of healthy animals are used as a source of $\operatorname{Pr}(\mathrm{PC})$ [12]. During the incubation phase, $\operatorname{PrP}(\mathrm{Sc})$ (abnormal form of the prion protein) forces $\mathrm{PrP}^{\mathrm{C}}$ to change conformation and aggregate. The sonication fragments these aggregates into small species that act as seeds able to promote further PrPC conversion. Therefore the ultrasounds act at the molecular level to determine the cleavage of the aggregates. Based on these reasons my hypothesis consist of using ultrasounds to counteract the new pandemic from COVID 19, which could weaken, the viral envelope constituted by a phospholipid bilayer of pericapsid and therefore through, for example, causing a conformational change of the spike S-glycoprotein receptor, which it uses to bind to the human ACE2 receptor to enter cells. $S$ glycoprotein is fundamental to mediate the membrane fusion required for virus entry and cell fusion. Ultrasound travels in the air, like COVID-19 does, so above all in closed environments, including homes, offices, supermarkets, schools, buses, subway, where the greatest contact occurs among the people, it could prevent the transmission from one individual to another in case of close contact, sneezing, coughing or breathing, thus avoiding contagion and the spread of the pandemic. The hypothesis is therefore to destroy the COVID-19 in the air contained in the microdroplets or in fine dust from environmental pollution, and also as soon as it comes into contact with the upper airways of humans, in such a way as to block or decrease its replication and therefore the viral load.

\section{Experimental Protocol}

Undergo the virus COVID-19 to a determined TCID50, to ultrasound at different exposure times: from a few seconds to a minute to test its immediate effectiveness of the ultrasound waves to inactivate the virus, then $10 \mathrm{~min}$, and $15 \mathrm{~min}$ (the latter, estimated minimum time for the infection to occur between individuals at a distance of less than one meter). Then $3 \mathrm{~h}, 6 \mathrm{~h}, 12 \mathrm{~h}, 24 \mathrm{~h}, 48 \mathrm{~h}$ and $72 \mathrm{~h}$ of exposure of the virus to ultrasound.

The Vero E6 cells (African green monkey kidney cells) [13] permissive to the SARS-CoV-2, will be respectively infected to a certain MOI, with the different samples virus treated with ultrasounds at the different times listed above. Then infected Vero E6 cells will be analyzed with MTT assay for to test the eventual inhibition of the cytopathic effect as well as a control of uninfected cells (mock infection), and a control virus of cells infected with the virus not treated with ultrasonic waves.

\section{Consequences of the Hypothesis and Discussion}

The transmission of infection can happen by main route, one is from the virus on the surfaces and other is infection from the

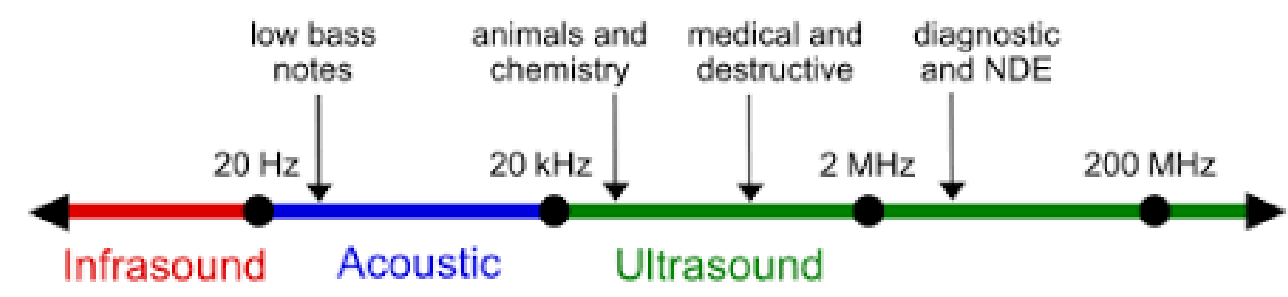

Figure 1: Diagram of the approximate frequencies corresponding to ultrasounds according to their field of application. Source Wikipedia. 
droplets from the sneeze and cough skin flakes [14-16]. Each of these processes generates aerosol droplets of different size and initial speed. When a person sneeze or coughs, talks loud, ejecting the droplets is released which is of $1 \mathrm{~mm}$ in diameter which falls on the ground within a minute [13], but the microdroplets remain through the air for several hours which are smaller than 10 micrometer. They are small and light drifting through the air and stays and does not drift from air for a period of time [17]. Have been observed that there is high risk of spread of infection in closed room or in a class room. For example, in a closed room of 10 people, if a person coughs once there is spread of 1,000 droplet with most of them fall on the ground in a minute whereas the microdroplet spreads in a very high volume for long period. Accordingly my hypothesis to use ultrasounds could be taken into consideration as they spread through the air where there are also the viral particles, but they are not harmful to human health, instead they could damage the molecular structures of pathogenic microorganisms including viruses and in particular potentially, also COVID-19 cause of the ongoing pandemic. The ultrasounds could therefore also be used to heal the environment in closed places because it has been seen that the virus remains in the air for several hours through microdroplets so in places where there are people it could act as a barrier to avoid contagion from a person to another in case of sneezing, coughing or just breathing but also from objects with virus to individuals. Ultimately, the use of ultrasound as an alternative and supportive means, even at relatively low costs, could prevent the further spread of COVID-19 and therefore block the pandemic curve, for a desirable recovery from the collapse of the world health system and from immense efforts of health workers. The application of this hypothesis could be of considerable impact and inspiration if developed and solve several health and socio-economic problems that unfortunately we are witnessing.

\section{References}

1. Wang C, Horby PW, Hayden FG, Gao GF (2020) A novel coronavirus outbreak of global health concern. Lancet 395: 470-473. [crossref]

2. Savarino A, Buonavoglia C, Norelli S, Di Trani L, Cassone A (2006) Potential therapies for coronaviuses. Expert Opinion Ther.Patents 16: 1269-128. [crossref]

3. J Oscanoa T, Romero-Ortuno R, Carvajal A, A Savarino A (2020) A pharmacological perspective of chloroquine in SARS-CoV-2 infection: An old drug for the fight against a new coronavirus? Int J Antimicrob Agents 56: 106078. [crossref]
4. Cortegiani A, Ingoglia G, Ippolito M, Giarratano A, Einav S (2020) A systematic review on the efficacy and safety of chloroquine for the treatment of COVID-19. J Crit Care 10: 57: 279-283. [crossref]

5. Liu J, Cao R, Xu M, et al. (2020) Hydroxychloroquine, a less toxic derivative of chloroquine, is effective in inhibiting SARS-CoV-2 infection in vitro. Cell Discov 6: 16. [crossref]

6. Gautret P, Lagier JC, Parola P, et al. (2020) Hydroxychloroquine and azithromycin as a treatment of COVID-19: results of an open-label non-randomized clinical trial. Int J Antimicrob Agents 105949. [crossref]

7. US Food and Drug Administration Report, 2000 US Department of Agriculture. Kinetics of microbial inactivation for alternative food processing technologies: ultrasound US Food and Drug Administration Report Published 2 June 2000.

8. S Condón-Abantoa, S Pedros-Garrido, M Marcen, V Ruiz, S Condón (2018) Synergistic effect of ultrasonic waves under pressure at mild temperatures (MTS) in yeast inactivation. International Journal of Food Microbiology 284: 56-62. [crossref]

9. Sarkinas A, Sakalauskiene K, Raisutis R, Zeime J, Salaseviciene A, Puidaite E, et al. (2018) Inactivation of some pathogenic bacteria and phytoviruses by ultrasonic treatment. Microb Pathog 123:144-148. [crossref]

10. Galina Matafonova , Valeriy Batoev (2020) Dual-frequency ultrasound: Strengths and shortcomings to water treatment and disinfection Laboratory of Engineering Ecology, Baikal Institute of Nature Management, Siberian Branch of Russian Academy of Sciences, Ulan-Ude, Russia Water Research 182: 116016.

11. Saborio GP, Permanne B, Soto C (2001) Sensitive detection of pathological prion protein by cyclic amplification of protein misfolding. Nature 411: 810-813.

12. Giaccone G, Moda F (2020) PMCA Applications for Prion Detection in Peripheral Tissues of Patients with Variant Creutzfeldt-Jakob Disease. Biomolecules 10: 405. [crossref]

13. Zhang Qinfen, Cui Jinming, Huang Xiaojun, Zheng Huanying, Huang Jicheng, et al. (2004) The life cycle of SARS coronavirus in Vero E6 cells. J Med Virol 73: 332-337. [crossref]

14. Lin L, Lu L, Cao W, Li T (2020) Hypothesis for potential pathogenesis of SARSCoV-2 infection--a review of immune changes in patients with viral pneumonia. Emerg Microbes Infect 9: 727-732. [crossref]

15. Morawska L (2006) Droplet fate in indoor environments, or can we prevent the spread of infection?. Another type of spread through closed personnel contact within the home, community centers, daycares, school classroom, University through the microdroplets. Indoor Air 16: 335-347. [crossref]

16. Barker J, Stevens D, Bloomfield SF (2001) Spread and prevention of some common viral infections in community facilities and domestic homes. J Appl Microbiol 91: 7-21. [crossref]

17. Fahad Al Qahtani, Modi Fahd Al Qahtani, Ahad Fahd Al Qahtani, Nagesh Bhat J (2020) Family Microdroplets are more infectious of COVID-19 spread in a closed door. Med Prim Care. 9: 3776-3777. [crossref]

\section{Citation:}

Norelli S (2021) Could Ultrasound Inactivate COVID-19 in the Environment and in the Air, Thus Preventing the Spread of the Pandemic? Internal Med Res Open J Volume 6(3): 1-3. 